\title{
LE SYSTÈME DES PASSIONS DANS LA TRAGÉDIE LULLYSTE. L'EXEMPLE DE LA FUREUR
}

\author{
CÉLINE BOHNERT \\ Université de Reims Champagne-Ardenne
}

\section{SYSTEM OF PASSIONS IN LULLY'S TRAGEDIES EN MUSIQUE. THE EXAMPLE OF FURY}

\begin{abstract}
What is the dramatic action in Lully and his librettists' "tragédies en musique"? We argue that it consists in the necessary deployment of a set of passions carefully articulated, according to an initially euphoric conception - some passions guarantee the world's harmony - that grows darker as the genre finds its autonomy in relation to the spectacular context from which it emerged.
\end{abstract}

Keywords: tragédie en musique, opera, dramaturgy, Lully, Quinault, passion, action, fury, rules, necessity

Mots clés : tragédie en musique, opéra, dramaturgie, Lully, Quinault, passion, action, fureur, règles, nécessité

Selon Pierre Perrin, l'un des inventeurs du théâtre lyrique français, « la fin du Poète lyrique " est "d'enlever l'homme tout entier » en touchant " en même temps l'oreille, l'esprit et le cœur : l'oreille par un beau son [...], l'esprit par un beau discours et par une belle composition de musique bien entreprise et bien raisonnée, et le cœur en excitant en lui une émotion de tendresse ${ }^{1} »$.

Sur ce pied[, précise-t-il,] j’ai tâché de faire mon discours de musique beau, propre au chant et pathétique : et dans cette vue j'en ai toujours choisi la matière dans les passions tendres, qui touchent le cœur par sympathie d'une passion pareille, d'amour ou de haine, de crainte ou de désir, de colère, de pitié, de merveille, etc., et j'en ai banni tous les raisonnements sérieux et qui se font dans la froideur, et même les passions graves, causées par des sujets sérieux, qui touchent le cœur sans l'attendrir².

1 Perrin, P. Recueil des paroles de musique. In Auld, L. E. (1986) : The Lyric Art of Pierre Perrin, Founder of French Opera, Henryville, Ottawa et Binningen : Institute of Medieval Music, Institut de Musique Médiévale et Institut für Mittelalterliche Musikforschung, t. III, p. vi. Pour l'analyse de ce passage, voir Kintzler, C. (1991) : Poétique de l’opéra français de Corneille à Rousseau. Paris : Minerve, « Les voies de l'Histoire ", pp. 360 sq. Pour le lien entre les passions et le chant, Naudeix, L. (2004) Dramaturgie de la tragédie en musique (1673-1764). Paris : Champion, pp. 40-53. Et pour les débats sur la moralité de l’opéra, en particulier la représentation des passions, Guyon-Lecoq, C. (2002) : La Vertu des passions. L'esthétique et la morale au miroir de la tragédie lyrique (1673-1733). Paris : Champion.

2 Perrin, P. (1986), ibidem. Tous les textes cités dans cet article ont été modernisés. 
Le théâtre chanté est ainsi défini par la tendresse en un sens large qui rejoint la notion de pathos, y compris dans ses excès. Les détracteurs de l'opéra que sont Saint-Evremond et Nicolas Boileau ne diront pas autre chose : le chant est vraisemblable voire «naturel » lorsqu'il sert l'expression de passions intimes - «tendres » : le terme est retenu systématiquement. Saint-Evremond affirme en effet qu'

il y a des choses qui doivent être chantées, il y en a qui peuvent lêtre sans choquer la bienséance, ni la raison. [...] les passions tendres et douloureuses s'expriment naturellement par une espece de Chant ; l'expression d'un amour que l'on sent naitre, l'irrésolution d'une âme combattuë de divers mouvemens, sont des matieres propres pour les Stances, et les Stances le sont assez pour le Chant ${ }^{3}$.

Soulignons que, dans l'esprit de Saint-Evremond, la musique est seconde : elle convient aux stances, qui, elles-mêmes, conviennent à l'expression des " mouvements » intérieurs causés par l'amour ou par l'irrésolution (amoureuse ?). Contraint de s'essayer à l'opéra, Boileau s'accordera avec Saint-Evremond pour lier la notion de tendresse à l'expression des passions privées; mais il la réduira à l'un de ses registres, le registre doux, ramené péjorativement au doucereux 4 .

Pour ces trois auteurs, la tendresse ainsi définie est "le partage du Chant ${ }^{5}$ ", son territoire propre, distingué d'un espace qu'ils désignent de manières différentes. La scène lyrique exclut ce que Perrin nomme les « raisonnements sérieux » et « les passions graves » (les passions qui, lorsqu'elles sont représentées, ne suscitent pas un effet pathétique "par sympathie d'une passion pareille »). Pour Saint-Evremond, le chant ne convient pas à l'expression d'une pensée rationnelle ; il ne saurait être employé pour ce qui est de l'ordre de l'organisation, du projet, de l'échange construit, qu'il s'agisse de paroles ou d'actes :

Tout ce qui est de la conversation et de la conférence, tout ce qui regarde les intrigues et les affaires, ce qui appartient au conseil et à l'action, est propre aux Comédiens qui récitent, et ridicule dans la bouche des Musiciens qui le chantent ${ }^{6}$.

Nulle délibération en chanson, nul acte de volonté, et, partant, aucune action véritable. Cette idée sous-tend sans doute le jugement expéditif qui conclut sa lettre sur les opéras :

Je comprendrai les plus grands défauts de nos Operas en peu de paroles : on y pense aller à une représentation, et l'on n'y représente rien ; on y veut voir une Comédie, et l'on n'y trouve aucun esprit de Comédie?

3 Saint-Evremond, "Sur les opéras. À monsieur le duc de Bouquinquant ", Euvres en prose, éd. René Ternois, Paris, Marcel Didier, 1966, t. III, p. 153.

4 Sur la notion de douceur, voir La Douceur en littérature de l'Antiquité au XVIIe siècle, éd. H. Baby et J. Rieu, Classiques Garnier, Paris, 2012, en particulier l'étude de A. Fiaschi-Dubois, « De la douceur dans la musique française du XVIIe siècle. Une catégorie esthétique emblématique d'un bon goût français qui s'opposerait au "picquant » des Italiens », p. 405-433.

5 Saint-Evremond, «Sur les opéras » éd. cit., p. 153.

Ibidem.

7 Ibid., p. 164. 
"L'on n'y représente rien » : non qu'il n'y ait rien à voir, bien au contraire, mais le spectacle, aussi fastueux soit-il, ne s'ordonne pas en une action : l'opéra ne repose pas sur une mimésis dramatique aux yeux de Saint-Evremond.

De prémisses assez proches, Boileau tire un dégoût comparable et une conclusion différente :

[...] on ne peut jamais faire un bon opéra, parce que la musique ne sauroit narrer; que les passions n'y peuvent être peintes dans toute l'étendue qu'elles demandent ; que d'ailleurs elle ne sauroit souvent mettre en chant les expressions vraiment sublimes et courageuses ${ }^{8}$.

De nouveau, l’opéra est donné pour une forme dramatique imparfaite par essence : « la musique ne sauroit narrer »- il faut entendre qu'elle ne peut mettre en ordre des actions. Or on sait que l'agencement de l'intrigue (muthos) constitue l'élément primordial dans la définition des genres dramatiques aux yeux des théoriciens du théâtre régulier ${ }^{9}$.

Sur ce point, les deux critiques sont d'accord. Mais Boileau est plus radical que Saint-Evremond en ce qui concerne l'expression des passions. Quelle est cette " étendue " que "demand[e] » la peinture des passions et qui manque au chant ? Veut-il dire que certaines passions ne peuvent être chantées (c'était l'idée de Perrin) ou que les passions ne sauraient être rendues dans toute leur intensité par le chant ? La seconde idée est nouvelle. Dans ce second cas, le territoire de la musique est restreint aux passions douces ou tendres en suivant le sens moderne de tendresse. Enfin Boileau ajoute une dernière attaque : non content de restreindre la nature de la représentation musicale (des états et non des actions) et l'intensité des états représentés, il met encore en cause la possibilité même d'une poésie propre au chant qui soit une véritable poésie. Les expressions «vraiment sublimes et courageuses » échappent à la musique. Le recours à un terme moral, " courageuses », jette sur les paroles de musique un discrédit absolu : pour Boileau, la grandeur poétique est affaire d'éthique autant que d'esthétique. Aussi séduisantes soient les paroles chantées, elles ne seront jamais que jolies : privées du socle éthique de la poésie, elles ne peuvent prétendre à la beauté.

On le voit, ces deux détracteurs de la scène lyrique révoquent en doute la possibilité même d'un drame en musique. Accordée à l'expression de la tendresse, la musique ne peut (en revanche, dirait Saint-Evremond ; pour cette raison même, ajouterait Boileau) servir le déploiement d'une intrigue dramatique. Ils posent ainsi une question fondamentale.

De fait, contrairement à ce qui se passe sur la scène déclamée, la parole sur la scène lyrique de la fin du XVII ${ }^{e}$ siècle est souvent moins action qu'exposé des motifs de l'action. Au temps de Lully, les personnages lyriques sont pris dans une forme de passivité paradoxale : d'un côté Cadmus, Thésée et Hercule réalisent sur scène des exploits héroïques, de l'autre ils sont essentiellement les bénéficiaires de la bienveillance des dieux et n'ont - presque - qu’à exposer leurs désirs pour les voir réalisés. Ces héros semblent moins définis par leurs actions que par les mouvements qui les traversent et

8 CEuvres complètes (1870-1873), éd. Charles-Antoine Gidel, Paris : Garnier frères, t. III, pp. 93-94.

9 Šuman, Z. (2013) : «Vraisemblance dans Héraclius de Pierre Corneille : théorisation de la crédibilité », in Filologické studie 2013. Medium et Scopus. Jazyky literatury v edukaci a komunikaci. Praha : Karolinum, pp. 71-78. 
par leur nature héroïque, qui suffit à leur attirer les grâces du ciel. Les livrets de Quinault donnent à voir de nombreux moments d'hésitation dans lesquels l'action est suspendue par le déchaînement des passions. Loin d'en conclure avec Saint-Evremond et Boileau que la notion de drame musical est un monstre théorique, on voudrait approcher la nature d'intrigues fondées sur l'expression musicale des passions, où la logique des actions s'articule à un système dynamique des affects.

\section{Un système de passions : I'organisation fonctionnelle des affects}

Alors qu'Aristote définit la tragédie comme un système de faits (Poétique, chap. 6, 50a15), la tragédie en musique se constitue, elle, comme un système de passions. On peut qualifier ce système de dramatique sans renoncer à ce qui fonde la notion : cet agencement, quoique de nature différente (agencement de passions et non d'actions) suit la logique impérieuse des principes débattus par les théoriciens classiques. Ainsi de la vraisemblance : Catherine Kintlzer, on s'en souvient, s'est appuyée sur l'analyse de cette notion pour montrer que la tragédie en musique est bien un genre dramatique, articulé à son double déclamé suivant un principe de parallélisme inversé ${ }^{10}$. Buford Norman, de son côté, souligne la spécificité de « la conception de l'action et de l'intrigue [lyriques], qui mettent parfois plus l'accent sur le contenu émotionnel de chaque scène que sur la succession des événements ${ }^{11}$ ». La vraisemblance de la scène lyrique selon B. Norman est d'ordre émotionnel : les réactions des personnages à des situations relevant d'une vraisemblance dite extraordinaire ou merveilleuse constituent le fil directeur du drame. À quelles logiques obéit alors le déploiement des intrigues ? Dans le cas de Pierre Perrin, on peut mettre en lumière une architecture des passions à l'échelle de l'acte : $L a$ Mort d'Adonis, la première tragédie en musique française, se compose de cinq tableaux passionnels successifs ${ }^{12}$. L'écriture de Lully et de ses librettistes est plus ample : les passions se déploient chez eux en grands mouvements qui, outrepassant la limite des actes, obéissent à un dessein général dont les éléments se répondent, en un tout unifié et fortement architecturé. Ainsi le déploiement concerté des passions détermine-t-il l'enchaînement d'actions susceptibles de les provoquer, sans doute plus que le contraire. Le cas de la fureur, cette passion paroxystique, permet de mettre en lumière cette hiérarchie qui inverse les données du théâtre parlé.

On peut bien sûr souligner l'intérêt purement matériel du tableau de la fureur, comme le remploi de décors, et insister sur l'efficacité spectaculaire d'une scène à faire : les parodistes ne s'en privent pas. Ils modélisent la création lyrique comme une addition d'éléments juxtaposés : Quinault disposerait au petit bonheur un petit nombre d'items,

10 Poétique de l'opéra français de Corneille à Rousseau, op. cit.

11 Norman, B. (2009) : Quinault, librettiste de Lully. Le poète des grâces, trad. Thomas Vernet et Jean Duron. Wavre : Mardaga, p. 41 ; du même, "Actions and Reactions : Emotional Vraisemblance in the Tragédie lyrique », Cahiers du Dix-septième siècle, 3 (1), 1989, pp. 141-154.

12 Bohnert, C. " La poétique des paroles de musique selon Pierre Perrin : l'exemple de La Mort d'Adonis ». In Goulet, A.-M. - Naudeix, L. (2010) : La Fabrique des paroles de musique en France à l'âge classique. Wavre : Mardaga, pp. 133-159. 
toujours les mêmes, puisés dans un magasin fort limité13. Mais l'observation des livrets montre que les moments de fureur apparaissent après un savant travail de préparation. L'ordre des passions suit une logique concertée. Notons d'abord que la représentation scénique de la fureur est presque toujours précédée par l'effroi qu'elle suscite : l'idée même du furieux suffit à faire trembler les personnages qui évoquent son image. Plus encore, la place des grandes scènes de fureur dans les livrets mis en musique par Lully amène à reconnaître en cette passion un élément fonctionnel du drame. Ces scènes se trouvent généralement en fin d'acte : elles constituent une ponctuation avant un rebond vers le mouvement suivant : épiphanie de Mars (III, 7) puis de Junon (IV, 7) dans Cadmus et Hermione; mer déchaînée par une Thétis en fureur ( $\mathrm{I}, 8)$ dans Alceste. Plus précisément, c'est l'acte III qui, dans de nombreux livrets, est le moment de la furie dans ses formes les plus spectaculaires. Dans Cadmus et Hermione, dont la composition suit un mouvement encore un peu mécanique, l'acte III se termine par une explosion de fureur de Mars, apaisé à l'acte suivant ; de la même manière, l'acte IV s'achève sur la colère de Junon, dont le parti de Cadmus viendra à bout. Alceste assouplit ce schéma : chaque acte est le lieu d'une crise (enlèvement, guerre, sacrifice) dont l'acmé est atteinte juste avant la fin de l'acte, qui amène un élément d'apaisement : Éole calme les flots (acte I), Apollon indique une voie de salut pour Admète (acte II), Diane ouvre la voie des enfers à Hercule (acte III). L'acte IV, lui, montre un apparent triomphe d'Hercule, qui ne sera effectif que lorsque le héros se sera vaincu lui-même. Si les trois premiers actes dessinent trois courbes distinctes, dont le dernier moment prépare l'acte suivant, les actes IV et V développent un dernier renversement, plus ample et paradoxal celui-là. La satisfaction de l'amour, malgré les apparences, constitue en réalité une crise pour le héros, amené à dépasser ses sentiments. Dans les livrets suivants, la composition des passions se complexifiera, en dessinant des courbes plus amples. Dans Thésée, l'acte I voit se déchaîner les fureurs de la guerre apaisées lors du divertissement : les prêtresses implorent Minerve de protéger toujours Athènes (I, 9). Il ne sera plus question de combats par la suite. C'est que d'autres fureurs sont à venir : celles de Médée, auxquelles la guerre ne sert que de préambule et peut-être de métaphore. Comme souvent, elles ont été annoncées bien avant que d'éclater : alors que le premier moment, celui des combats, est près de se terminer, Égée évoque la puissance de la magicienne ; il lance ainsi la seconde grande ligne, celle que suivra la tragédie par la suite $(I, 8)$.

Que Médée en fureur s'arme, menace, tonne,

Il faut que ma main vous couronne

Quand il m'en coûterait et l'Empire et le jour.

Un grand cœur qui se sent animé par l'amour

Ne doit jamais trouver de péril qui l'étonne.

J'atteste Minerve à vos yeux,

J'atteste le Maître des cieux,

Et sa foudroyante justice ${ }^{14} \ldots$

13 Leblanc, J. (2014) : Avatars d’opéras. Parodies et circulation des airs chantés sur les scènes parisiennes (1672-1745). Paris : Classiques Garnier, «Lire le XVIIe siècle. Série Musique et littérature ».

14 Toutes les citations sont prises dans Quinault, Ph. (1999) : Livrets d'opéra. Présentés et annotés par Buford Norman, Toulouse : Société d'études classiques, $2 \mathrm{t}$. 
Il faut attendre la fin de l'acte II, et la révélation de l'amour que se portent mutuellement Thésée et Églé, pour que Médée bascule effectivement dans la fureur : c'est la grande scène qui commence par " Dépit mortel, transports jaloux ", moment charnière vers l'acte III où la magicienne commencera d'accomplir sa vengeance, de transformer l'énergie pure de la fureur en une série d'actions soumises à un projet ${ }^{15}$. Après le divertissement des habitants des Enfers qui couronne l'acte III, l'acte IV se déroule dans l'espace infernal ; celui-ci en vient à céder la place à une île faussement enchantée, qui est pour Églé un lieu de torture : la fureur s'extériorise en principe spectaculaire, elle donne naissance à un personnel puis à un espace adéquats, avant d'être de nouveau intériorisée dans une situation psychologique extrême. Le vaste mouvement que dessinent les fureurs de Médée connaît par la suite deux rebonds : un premier, intense mais de faible ampleur, où la magicienne convainc Égée d'empoisonner son fils $(\mathrm{V}, 3)$; mais son projet est rapidement contrecarré. Un second, plus violent et tout aussi bref, amène la destruction de l'espace enchanteur fondé par Médée pour lui substituer un nouveau palais. Lully et Quinault ménagent ainsi une montée progressive vers le déchaînement de la fureur (acte III), puis un apaisement tout aussi minutieusement dessiné, conclu après un dernier sursaut. En un dessin plus simple, Proserpine déploie également une grande ligne allant d'une harmonie nostalgique (Cérès se plaint de ne plus être aimée de Jupiter, tout en savourant le bonheur de la maternité) à un retour à la tendresse qui passe par l'état extrême de la fureur vengeresse. L'enlèvement de Proserpine à la fin de l'acte II amène la fureur de Cérès à la fin de l'acte suivant; à l'acte IV, la colère de la jeune femme aux enfers semble mimer celle de sa mère en un écho atténué, suivant une courbe de moindre ampleur : son retour au calme précède et annonce celui de sa mère, dont le basculement de la douceur à la rage, puis le retour à soi tracent la courbe principale.

Les deux dernières pièces coécrites par Lully et Quinault échappent à ce modèle. La fureur de Roland, moment clé du drame, se déploie sur deux actes : la rage du héros atteint son paroxysme à la fin de l'acte IV, et l'acte suivant dessine une ligne descendante, jusqu’à sa guérison. Seule Armide, qui semble radicaliser l'essai de Roland, est polarisée vers le spectacle de la fureur.

Dans les autres pièces, si la conclusion spectaculaire n'est pas toujours heureuse (lorsque c'est le cas il s'agit d'un mariage ou de la célébration du héros rendu à lui-même), du moins repose-t-elle toujours sur une remise en ordre - $\mathrm{y}$ compris dans la pièce qui approche le plus du tragique métaphysique tel qu'il est défini depuis le romantisme, Atys : la métamorphose du héros sacrifié réintègre la douleur de Cybèle, cause de chaos cosmique, dans l'ordre du monde. De ce fait, elle transforme la nature de la passion et la soumet à une temporalité ordonnée : la douleur devient deuil. Pourvue d'un signe qui en constitue un mémorial, exprimée par des gestes rituels, la souffrance trouve sa place dans l'harmonie universelle et s'inscrit dans la succession des temps. De même, le dénouement de Phaéton repose sur la nécessité de refonder un ordre mis à mal par l’orgueil du jeune homme.

Ainsi la fureur dans les tragédies en musique lullystes constitue-t-elle un élément fonctionnel du drame : ponctuation, pivot et contrepoint, elle est un moment à dépasser.

15 Leblanc, J. « Poétique de la vengeance dans l'opéra lullyste : une affaire de femmes ». In Bohnert, C. - Borderie, R. (2013) : Poétiques de la vengeance : de la passion à l'action (XVIe-XXIe siècles). Paris : Classiques Garnier, «Rencontres. Série Littérature générale et comparée », p. 63. 
Déchaînement des éléments, cérémonie macabre ou spectacle d'un déchirement intérieur, elle prépare l'épiphanie conclusive et en fournit l'image inversée (dans le cas des dénouements heureux) ou, pour Atys, l'antithèse chaotique. Les tragédies en musique lullystes obéissent ainsi à une organisation concertée des passions. Propres à chaque pièce, l'agencement des lignes de force répond néanmoins à des principes communs : des scansions nettes notamment, structurées autour de scènes fortes habilement distribuées dans l'œuvre et intimement liées les unes aux autres par la logique interne des passions.

\section{La nature dramatique des passions}

Car si, à l'échelle de l'œuvre, la fureur interfère avec d'autres affects, cette passion est également dotée d'une dynamique propre, de nature dramatique. Le trouble furieux obéit à une logique qui détermine son déploiement.

En première approche, la fureur peut bien sûr être caractérisée comme un état psychologique. Elle apparait alors comme la grimace de la tendresse : elle en offre l'image dégradée tout en conservant ses traits principaux. Données toutes deux pour irrépressibles, ces passions mêlent indissociablement douceur et souffrance. Poussées à leur extrême degré, elles sont source de plaisir - celui du bien d'autrui ou celui, monstrueux et pervers, de sa souffrance. Mais l'harmonie, l'union et la paix que refonde l'accord des cœurs s'inverse en destruction, en ravage et en discorde du côté de la furie ${ }^{16}$. Toutes deux suivent un même mouvement inéluctable d'extériorisation. La pudeur des amants, le secret gardé sur les sentiments d'autrui, ne sont qu'un premier moment ; la passion partagée s'accomplit dans la cérémonie publique du mariage, signe autant que facteur d'harmonie universelle. Symétriquement, la fureur qui couve d'abord dans l'intimité du cœur en vient toujours, très vite, à exploser, emportant tout sur son passage. Certains traits de la première sont ainsi inversés dans la seconde (la soumission du parfait amant à l'intérêt de l'aimé s'inverse en désir de possession ou de destruction de lềtre chéri chez le furieux ${ }^{17}$ ), tandis que d'autres sont non pas retournés, mais dégradés : si l'amour héroïque et sage doit mener au partage et à l'union, la fureur vise la contagion, elle cherche à se répandre ${ }^{18}$. Les personnages déchirés prétendent faire vibrer l'univers à l'unisson de leur douleur.

C'est sans doute ce qui permet de reconnaitre en ces deux formes de la passion deux principes cosmologiques. Le terme fureur est employé de manière massive comme une métaphore des troubles du monde : guerre, tempête, orage... le cosmos devient un grand tout soumis au tiraillement de passions contraires - les unes garantissant son harmonie, les autres menaçant son ordre. La passion, quelle qu'elle soit, est essentiellement appréhendée au départ comme un trouble ; mais l'amour vertueux mène à la félicité, il fonde un ordre renouvelé, radieux, là où la fureur, malgré les jouissances qu'elle provoque, n'a d'autre issue que le chaos. Un accord intime, consubstantiel, est ainsi établi

16 Proserpine, III, 7, Cérès : «Après un si sensible outrage, / Mon cœur désespéré s'abandonne à la rage. / Du monde trop heureux je veux troubler la paix : / Brûlons, ravageons tout, détruisons mes bienfaits. "

17 Persée, IV, 3, Phinée : "L’Amour meurt dans mon cœur, la rage lui succède. / J'aime mieux voir un monstre affreux / Dévorer l'ingrate Andromède, / Que la voir dans les bras de mon rival heureux. "

18 Proserpine, II, 8, Cérès : "Que tout se ressente / De la fureur que je sens. » Cet axiome se traduit sur le plan de l'écriture musicale par les duos des furieux, comme ceux de Célénus et Cybèle dans Atys: très souvent, un personnage en emporte un autre dans sa rage. 
entre le for intérieur, le monde social et le monde naturel. Cet accord n'est pas seulement symbolique ou métaphorique, l'effet miroir révèle les structures profondes qui régissent les puissances tapies dans les cœurs et dans la nature. Ce sont les mêmes, de sorte que les mouvements qui bouleversent le cœur affectent immédiatement la nature - l'inverse n'est pas vrai. Tempête, orage, cataclysme extériorisent les affects des dieux, en réponse à ceux des humains.

Enfin l'amour vertueux et la fureur sont dotées de temporalités distinctes. Si le premier est caractérisé par la constance, la seconde est, jusqu’à un certain degré, réversible. L'absence de réponse de l'être aimé déclenche le processus furieux. Mais la tendresse continue de parler au cœur de l'enragé, qui oscille de la vengeance au pardon. Déformation grimaçante de l'amour héroïque, et son double obscur, la fureur est un alliage impur d'amour et de rage, elle laisse parfois voir, comme par transparence, l'élan premier dont elle est l'inversion ${ }^{19}$. Opposée à la constance des héros tendres (avatar de l'équanimité propre à l'héroïsme épique) et au sentiment de l'immuabilité d'un ordre cosmique, la fureur, ainsi définie, est une passion éminemment dramatique : plus qu'un état, elle est un scénario psychologico-cosmique. L’amour sans réponse provoque le désespoir qui s'approfondit en rage : le furieux devient une menace vitale contre l'aimé, jusqu'à ce qu'un retour de tendresse signale le caractère inachevé de la métamorphose ${ }^{20}$.

\section{Passions et nécessité}

Ainsi, si Aristote définissait la dynamique tragique comme le renversement d'une situation extrême à son opposé, c'est le passage d'un état à un autre qui fonde la dynamique de la tragédie en musique - et cet état est indissociablement celui des choses et des êtres, qui forment un ensemble solidaire, successivement traversé de mouvements contraires.

Cette logique inhérente à la fureur rejoint les canons de la dramaturgie aristotélicienne. Le caractère irrépressible des passions fonde la nécessité de l'intrigue. Les recours opposés à la passion signalent cette dernière comme un élan irrésistable : la mort ou le sommeil, qui éteignent l'incendie intérieur sans lui apporter de solution ${ }^{21}$; ou encore la magie, dans le cas de Roland ; ou bien le rappel à l'ordre d'une divinité supérieure ${ }^{22}$. Le dépit, qui mène à l'indifférence, est sans cesse évoqué comme la seule issue possible à la passion. Mais cette voie n'est presque jamais pratiquée par les grandes âmes ${ }^{23}$. À deux reprises dans les douze livrets mis en musique par Lully les héros connaissent un sursaut intérieur qui leur permet de dépasser la fureur (et l'amour) : c'est le cas d'Alcide dans

19 Atys, V, 4, Cybèle : « Atys, je vous ai trop aimé. / Cet amour, par vous-même en courroux transformé, / Fait voir encor sa violence. / Jugez, ingrat, jugez en ce funeste jour, / De la grandeur de mon amour / Par la grandeur de ma vengeance. "

20 Atys, III, 4, les Songes Funestes : «L'amour qu'on outrage / Se transforme en rage. »

21 Dans Persée Mérope ne sort du cycle de la fureur que par la mort ; et le sommeil est le recours que Mercure propose aux Gorgones contre les affres de la fureur ; la fée Logistille endort Roland dans la pièce éponyme.

22 Jupiter ordonne à Junon de renoncer à sa colère dans Cadmus, Isis et Persée.

23 Il n'est pratiqué que par Ascalaphe dans Proserpine. 
Alceste, de Junon dans Isis, à l'incitation de Jupiter ${ }^{24}$. Il n'est de paix (mais non d'immobilité) que dans l'accomplissement des passions heureuses. Ailleurs, l'ébranlement des passions, de la fureur tout particulièrement, ne trouve aucun frein intérieur.

Catherine Kintlzer signalait la conception cartésienne qui sous-tend ces livrets où les passions relèvent dans leurs manifestations, leur déroulement, leur symptomatologie, de ce qu’elle désigne comme une mécanique :

Il n'est question que de mouvements, d'agitations, d'excitations et d'ébranlements : vocabulaire qui n'est nullement employé en un sens métaphorique, mais renvoie à la matérialité du corps et aux effets par lesquels il manifeste son étroite liaison avec lâme. L'émotion des esprits animaux, on le sait, est pour Descartes l'agent nécessaire du phénomène passionnel. Parce qu'elles se manifestent à travers des symptômes matériels, parce que l'âme n'a aucun pouvoir direct sur leur surgissement et leur déroulement, enfin parce que, nonobstant cette impuissance, l'âme a l'illusion de les sentir comme en elle-même, il faut appeler ces émotions des passions ${ }^{25}$.

La matérialité des passions renvoie à leur accointance avec le corps, voire à leur nature corporelle. Si Descartes défend l'idée d'un pouvoir de la volonté sur les passions, il n’en souligne pas moins, de fait, l'autonomie des passions par rapport à l'âme, qui ne saurait leur donner naissance ${ }^{26}$.

Cette conception se manifeste dans les livrets par l'omniprésence de métaphores spatiales, en particulier dans l'emploi des verbes suivre et fuir. Si les personnages raciniens disent "J'aime", avec tout ce que cela signifie de stupeur ou de douceur, ceux des librettistes de Lully, en une spatialisation des affects, déclarent « suivre l'Amour » ou le fuir, sans possibilité de résister à cette aimantation.

La personnification des affects constitue également un procédé littéraire révélateur. Elle tend à dédoubler la scène lyrique, structurée en profondeur par le modèle du théâtre dans le théâtre : les personnages assistent, généralement en spectateurs impuissants, aux mouvements que les passions déclenchent en eux. A la limite, les caractères lyriques deviennent les simples supports de ce drame au second degré. Ainsi dans Thésée, Médée se voit-elle être Médée :

Mon frère et mes deux fils ont été les victimes

De mon implacable fureur ;

J'ai rempli l'univers d'horreur ;

Mais le cruel Amour a fait seul tous mes crimes. (I, 1, MÉDÉE, v. 317-320)

\section{Ou encore :}

Mon dépit, tu le sais, dédaigne de se plaindre

Il est difficile à calmer,

${ }^{24}$ Isis, V, 3, Jupiter : «Quoi ! le cœur de Junon, quelque grand qu'il puisse être, / Ne saurait triompher d'une injuste fureur? » puis Junon : « Nymphe, je veux finir votre peine cruelle ; / Que la Furie emporte aux Enfers avec elle / Le trouble et les horreurs dont vos sens sont saisis. »

25 Kintzler, C., Poétique de l'opéra français de Corneille à Rousseau, op. cit., p. 111.

26 Kambouchner, D. (1995) : L'homme des passions : commentaires sur Descartes. Paris : Albin Michel, «Bibliothèque du Collège international de philosophie », t. II., pp. 16-146. 
S'il venait à se rallumer,

Il faudrait du sang pour l'éteindre. (v. 330)

On trouve là un souvenir du "Medea nunc sum » de Sénèque, mais revêtu d'une signification nouvelle, car l'idée prend place dans un dispositif dramatique tout différent. Églé, d'ailleurs, ne s'exprime pas autrement :

Malgré Médée et sa vengeance

Mon amour fera son devoir. (III, 3)

De livret en livret, la métonymie dresse une scène seconde où s'affrontent des entités opposées. Les personnages lyriques dépendent extérieurement des actes des dieux de l'Olympe (ou de magiciennes), intérieurement de forces qui les agissent. La manie qu'ont les personnages de se désigner à la troisième personne, comme des objets offerts aux regards et définis par un universel consensus, renforce cette structure. Lorsqu'Égée remercie Médée, il s'adresse à la magicienne à la troisième personne, brandissant son nom comme un talisman aux yeux Médée même - et secondairement mais indissociablement aux yeux de l'univers : " Médée et son art redoutable / Ont gardé ce palais contre mes ennemis » (I, 2, v. 344-345). Dans les livrets lullystes, l'introspection s'accomplit par la lumière que jette un regard impersonnel sur le monde intérieur : une instance surplombante prend acte de ce qui s'agite en l'être profond des personnages. Les réécritures des livrets au XVIII siècle, en passant de la troisième personne aux personnes de l'interlocution, gommeront ce qui est plus qu'un stylème : le révélateur d'une pensée de la nature des passions. Le je est ici une instance extérieure et omnisciente ; une entité étrangère aux passions et qui assiste à distance au spectacle que lui donnent ces dernières. Cette ostentation de soi nous semble aussi un héritage des fêtes de cour : parades, entrées de villes, ballets. Le souverain et les grands s'y montraient sous tel ou tel masque (la lune, Roland, une Furie), eux-mêmes et cependant autres. Ce dédoublement, habilement exploité par Benserade dans les vers de ballet, crée autre chose encore, au-delà du frottement subtilement discordant entre la personne et le personnage : une troisième réalité, symbolique, émerge, qui n'est autre que la royauté idéale. Les fêtes publiques constituaient un spectacle que la royauté (plus que le roi) se donnait à elle-même, dans laquelle elle était proprement instituée sur le plan symbolique. Nous croyons déceler le souvenir de cette construction spectaculaire dans cette forme de spectacle de cour particulière qu'est la tragédie en musique à sa naissance.

Entée sur une conception que l'on dira cartésienne des passions, la fureur est donc moins un état qu'un processus. Son déploiement et ses oscillations, qui se traduisent dans l'intrigue par telle ou telle action (la trahison de l'aimé, par exemple, dénoncé à une entité supérieure), décident des moments du drame. Voilà qui contredit les parodistes : la méthode des librettistes ne consiste pas à agencer des éléments statiques vaguement reliés entre eux, suivant un catalogue de situations et d'humeurs. L'intrigue des tragédies en musique obéit à une nécessité interne qui est précisément celle des passions. Le caractère irrépressible de ces dernières rejoint les attentes de la théorie dramatique en fondant le caractère nécessaire de l'intrigue. 
Parlera-t-on pour autant de fatalité ? Jusqu'avant Amadis, on évoquerait plutôt une programmation des êtres. Les tragédies en musique s'offrent comme le déploiement d'une tautologie : un héros est un héros... et un furieux est un furieux. La fureur, en effet, appartient à deux types de personnages. Les comparses, d'une part, comme Mérope et Phinée dans Persée ou Lycomède dans Alceste : le basculement dans la fureur est chez eux le fait d'un manque de grandeur, d'une nature imparfaite. En revanche, pour les grandes figures de furieuses (ce sont toutes des femmes, et des magiciennes), il s'agit d'un habitus, d'une disposition naturelle chez des êtres en sympathie immédiate avec les espaces infernaux. Mais la dichotomie de départ, très claire, entre les amants héroïques et les furieux, se défait dans les trois dernières pièces, Amadis, Roland et Armide, qui reposent sur une conception renouvelée, plus sombre, de la passion. Il n'y a plus ici deux camps distincts : d'un côté les passions vertueuses et les êtres d'exception, de l'autre les passions mauvaises déchaînées par des êtres infernaux. Les passions sont désormais déconnectées de la question de la valeur, ce qui jette le trouble sur des affects jusque-là montrés comme positifs.

Qu'est-ce qu'une action dans la tragédie lullyste ? On répondrait brièvement qu'il s'agit du déploiement nécessaire d'un ensemble de passions soigneusement articulées entre elles, suivant une conception d'abord euphorique du phénomène passionnel - certaines fondent l'ordre du monde - puis beaucoup plus sombre à mesure que le genre trouve son autonomie par rapport au contexte spectaculaire dont il est issu.

\section{BIBLIOGRAPHIE}

Bohnert, C. «La poétique des paroles de musique selon Pierre Perrin : l'exemple de La Mort d'Adonis ». In Goulet, A.-M. - Naudeix, L. (2010) : La Fabrique des paroles de musique en France à l'âge classique. Wavre : Mardaga, pp. 133-159.

Boileau, N. (1870-1873) : CEuvres complètes. Éd. Charles-Antoine Gidel. Paris : Garnier frères, t. III.

Guyon-Lecoq, C., (2002) : La Vertu des passions. L’esthétique et la morale au miroir de la tragédie lyrique (1673-1733). Paris : Champion, « Moralia».

Kambouchner, D. (1995) : L'homme des passions : commentaires sur Descartes. Paris : Albin Michel, «Bibliothèque du Collège international de philosophie », $2 \mathrm{t}$.

Kintzler, C. (1991) : Poétique de l'opéra français de Corneille à Rousseau. Paris : Minerve, "Voies de l'histoire. Culture et société ".

Naudeix, L. (2004) : Dramaturgie de la tragédie en musique (1673-1764). Paris : Champion, « Lumière classique ".

Leblanc, J. (2014) : Avatars d'opéras. Parodies et circulation des airs chantés sur les scènes parisiennes (1672-1745). Paris : Classiques Garnier, «Lire le XVIIe siècle. Série Musique et littérature ».

Leblanc, J. « Poétique de la vengeance dans l'opéra lullyste : une affaire de femmes ». In Bohnert, C. Borderie, R. (2013) : Poétiques de la vengeance : de la passion à l'action (XVI $-X X I^{e}$ siècles). Paris : Classiques Garnier, «Rencontres. Série Littérature générale et comparée ».

Norman, B. (2009) : Quinault, librettiste de Lully. Le poète des grâces, trad. Thomas Vernet et Jean Duron. Wavre : Mardaga, «Études du Centre de musique baroque de Versailles».

Norman, B. (1989) : «Actions and Reactions : Emotional Vraisemblance in the Tragédie lyrique », Cahiers du Dix-septième siècle, 3 (1), 1989, pp. 141-154.

Perrin, P. Recueil des paroles de musique. In Auld, L. E. (1986) : The Lyric Art of Pierre Perrin, Founder of French Opera. Henryville, Ottawa et Binningen : Institute of Medieval Music, Institut de Musique Médiévale et Institut für Mittelalterliche Musikforschung, t. III. 
Quinault, Ph. (1999) : Livrets d'opéra. Présentés et annotés par Buford Norman. Toulouse : Société d'études classiques, $2 \mathrm{t}$.

Saint-Evremond : «Sur les opéras. À monsieur le duc de Bouquinquant ». In Saint-Evremond (1966) : Euvres en prose, éd. René Ternois. Paris, Marcel Didier, t. III.

Šuman, Z. (2013) : "Vraisemblance dans Héraclius de Pierre Corneille : théorisation de la crédibilité », in Filologické studie 2013. Medium et Scopus. Jazyky literatury v edukaci a komunikaci. Praha : Karolinum, pp. 71-78.

\section{Céline Bohnert}

Département de Lettres modernes - CRIMEL (EA3311)

Université de Reims Champagne-Ardenne

57 avenue Pierre Taittinger - 51100 Reims - France

celine.bohnert@univ-reims.fr 